\title{
Estadiamento cirúrgico do câncer de colo de útero localmente avançado
}

\author{
Surgical staging of locally advanced uterine cervix cancer
}

\author{
Heitor Ricardo Cosiski Marana ${ }^{1}$,Francisco José Cândido dos Reis ${ }^{2}$, Jurandyr Moreira de Andrade ${ }^{3}$, \\ Omero Benedito Poli ${ }^{4}$, Daniel Guimarães Tiezzi ${ }^{5}$, Fábio Eduardo Zola ${ }^{6}$
}

\section{RESUMO}

Objetivo: verificar em que proporção o estadiamento cirúrgico difere do estadiamento clínico entre casos com carcinoma avançado do colo do útero e a porcentagem de casos com gânglios para-aórticos positivos neste grupo de pacientes. Métodos: estudo prospectivo descritivo no qual foram incluídas 36 pacientes com diagnóstico histológico de carcinoma de colo de útero considerados localmente avançados (estadios IB2, IIB, IIIAeB e IVA). Foram submetidas a estadiamento clinico conforme as recomendações da FIGO. Todas eram candidatas ao tratamento com quimioterapia neoadjuvante. A idade variou de 40 a 73 anos, com média de $56,2 \pm 7,9$ anos. O procedimento constou de linfadenectomia pélvica seguida de linfadenectomia para-aórtica se os linfonodos pélvicos fossem positivos ao exame intra-operatório. A abordagem da cavidade e linfadenectomia foram efetuados por via laparotômica ou laparoscópica, indicados aleatoriamente. Os casos foram comparados individualmente e para cada estadiamento clínico foram estabelecidos os respectivos achados cirúrgicos que foram considerados o padrão-ouro. Resultados: na fase de estadiamento clínico (EC) 7 casos foram classificados como IB2 (tumores com mais de $4 \mathrm{~cm}$ ), 22 casos como EC II e 7 casos ECIII. A avaliação cirúrgica modificou o estadiamento clínico da seguinte foram: em seis casos o estadio foi diminuído, e em 13 casos os achados levaram à elevação do estadio. Houve concordância em apenas 18 casos (50\%). Em seis casos (16,9\%) os linfonodos para-aórticos estavam comprometidos. Conclusões: o estadiamento clínico do carcinoma de colo de útero localmente avançado é incorreto em proporção alta dos casos. Esta divergência levaria a indicação de tratamento excessivo em alguns casos, mas cerca de um quarto das pacientes com gânglios para-aórticos positivos não seria adequadamente tratado com o tratamento padrão atual - radioterapia com quimiossensibilização que é dirigida ao controle locorregional da doença na pelve.

PALAVRAS-CHAVE: Neoplasias do colo uterino; Estadiamento de neoplasias; Metástase neoplásica; Gânglios

\section{ABSTRACT}

Purpose: to assess to what extent the surgical staging differs from the clinical staging among cases of advanced uterine cervix carcinoma, and also to assess the percentage of cases with positive para-aortic ganglia in this group of patients. Methods: this is a descriptive prospective study in which 36 patients with histological diagnosis of uterine cervix carcinoma considered locally advanced were included (stages IB2, IIB, IIIA and B, and IVA). The cases were submitted to clinical staging, according to FIGO criteria. All patients were to be treated with neoadjuvant chemotherapy. Age ranged from 40 to 73 years, with a mean of 56.2 \pm 7.9 . The procedure started with pelvic lymphadenectomy followed by para-aortic lymphadenectomy, in case the pelvic lymph nodes were positive on surgical examination. Examination of the abdominal cavity and lymphadenectomy were done either through laparotomy or laparoscopy, chosen at random. In each case, the clinical staging was compared to the surgical staging, considered the gold standard. Results: in the clinical staging (CS), 7 cases were classified as IB2 (tumors larger than $4 \mathrm{~cm}$ ), 22 cases as CSII and 7 cases as CSIII. The surgical assessment changed the clinical staging as follows: the stage was decreased in six cases, and increased in 13. There was agreement only in 18 cases (50\%). The para-aortic lymph nodes were affected in six cases. Conclusions: clinical staging of locally advanced uterine cervix carcinoma is incorrect in most of the cases. Such inconsistency may lead to excessive treatment in some cases, but about one fourth of the patients with positive para-aortic ganglia would not be adequately treated with the current standard treatment - radiotherapy with chemosensitization, which aims at the local regional control of the pelvic disease.

KEYWORDS: Cervix neoplasms; Neoplasm staging; Neoplasm metastasis; Ganglia

Trabalho realizado no Hospital das Clinicas da Faculdade de Medicina da Universidade de São Paulo - USP - Ribeirão Preto (SP), Brasil.

1 Médico Assistente do Hospital das Clinicas da FMRP-USP - Setor de Oncologia Ginecológica e Mastologia do Departamento de Ginecologia e Obstetrícia da FMRP - USP.

2 Professor Associado do Departamento de Ginecologia e Obstetrícia da Faculdade de Medicina de Ribeirão Preto, Universidade de São Paulo - USP Ribeirão Preto (SP) - Brasil.

3 Professor Titular do Departamento de Ginecologia e Obstetrícia da Faculdade de Medicina de Ribeirão Preto, Universidade de São Paulo - USP Ribeirão Preto (SP) - Brasil.

4 Professor do Departamento de Cirurgia e Anatomia da Faculdade de Medicina de Ribeirão Preto da Universidade de São Paulo.

5 Médico Assistente do Hospital das Clinicas da Faculdade de Medicina de Ribeirão Preto, Universidade de São Paulo - USP - Ribeirão Preto (SP) - Brasil - Serviço de Oncologia. Pós-Graduando - Departamento de Ginecologia e Obstetrícia da FMRP-USP.

6 Médico Assistente do Hospital das Clinicas da FMRP-USP - Setor de Oncologia Ginecológica e Mastologia; Pós Graduando - Departamento de Ginecologia e Obstetrícia da Faculdade de Medicina de Ribeirão Preto, Universidade de São Paulo - USP - Ribeirão Preto (SP) - Brasil.

Correspondência: Heitor R C Marana

Departamento de Ginecologia e Obstetrícia da Faculdade de Medicina de Ribeirão Preto da Universidade de São Paulo (FMRP-USP)

Avenida Bandeirantes 3900, $8^{\circ}$ andar - 14049-900 - Ribeirão Preto - SP - Fone (16) 3633-0216; Fax (16) 3633-0946

Recebido em: 9/5/2005

Aceito com modificações em: 21/11/2005

Rev Bras Ginecol Obstet. 2005; 27(12): 744-9 


\section{Introdução}

O câncer de colo de útero é o segundo tipo de câncer mais comum entre mulheres em todo o mundo, com cerca de 500.000 casos novos por ano dos quais, $80 \%$ são diagnosticados em países em desenvolvimento ${ }^{1}$. No Brasil estima-se a incidência em 22 casos para cada 100.000 mulheres, e se prevê a ocorrência de 20.690 casos novos em 2005, com expectativa de 8.000 mortes $^{2}$. Destes casos, estima-se que $43 \%$ se apresentem em estádios considerados localmente avançados (estádios III e IVA) ${ }^{3}$ e, são portanto candidatas a tratamento sistêmico.

O tratamento do câncer de colo de útero inicial é cirúrgico (estádios IA2, IB1 e casos selecionados no estadio IIA). Para as pacientes com tumores localmente avançados (estádios IIB, III e IVA) as alternativas são a radioterapia, a radioterapia com quimiossensibilização e a quimioterapia neoadjuvante seguida de cirurgia. A recomendação para o emprego da associação da quimio com radioterapia baseia-se em resultados de trabalhos recentes que apontam para melhoria da sobrevida global e do intervalo livre de doença ${ }^{4,5}$. Esta última alternativa de tratamento é, de fato, capaz de melhorar a eficiência da radioterapia no controle local e tem impacto positivo sobre a sobrevida. No entanto há duvidas sobre a sua eficiência no controle da doença metastática ${ }^{6-8}$. Por este motivo as pacientes com linfonodos para-aórticos comprometidos deveriam ser submetidos a esquemas de múltiplas drogas e com doses maiores empregando-se a quimioterapia neoadjuvante, que é eficiente para tratamento dos focos neoplásicos situados fora da pelve ${ }^{9}$.

Além disto o achado de gânglios pélvicos positivos no material obtido de cirurgia para casos com carcinoma inicial torna o prognóstico pior e é indicação para radioterapia pélvica. No entanto, sabe-se que a morbidade associada ao tratamento é muito aumentada nos casos em que se emprega radioterapia adjuvante após a cirurgia ${ }^{10}$ o que reforça a hipótese que a avaliação do estado dos gânglios no pré-operatório seria muito útil para seleção adequada do tratamento mesmo nos estádios iniciais.

As alternativas atuais para melhora da acurácia do estadiamento com inventário completo da expansão da doença para estruturas vizinhas e para os gânglios são a avaliação cirúrgica e o emprego de métodos de imagem - tomografia computadorizada $(\mathrm{CT})$, ressonância magnética $(\mathrm{RM})$ e positron emission tomography (PET). Todos os métodos de imagem mostram boa acurácia para avalia- ção do tamanho do tumor, detecção do envolvimento do paramétrio e vagina, e de gânglios com volume aumentado ${ }^{11}$. No entanto, a detecção de metástases em gânglios com volume normal e mesmo a avaliação correta da disseminação da doença na cavidade abdominal só podem ser efetuadas, atualmente, pela inspeção intra-operatória e histopatologia ${ }^{12,13}$.

Por estes motivos o estadiamento cirúrgico rotineiro para tumores localmente avançados tem sido adotado ${ }^{12,14}$ embora o impacto sobre a sobrevida não tenha sido analisado e a sua utilidade não tenha sido demonstrada em estudos controlados. Assim, os objetivos deste trabalho foram: avaliar a correlação entre o estadiamento clínico e o obtido pela cirurgia entre pacientes com carcinoma de colo localmente avançado e a porcentagem de casos com linfonodos para-aórticos com metástases. Como objetivo secundário avaliar a duração dos procedimentos e a morbidade a eles associada.

\section{Métodos}

Foram incluídas 36 pacientes submetidas à estadiamento clínico e cirúrgico para carcinoma de colo localmente avançado. Os critérios de inclusão foram: diagnóstico histológico de carcinoma de colo, condições clínicas para tratamento quimioterápico com cisplatina e presença de condições locais, verificadas durante a cirurgia, para dissecção de gânglios pélvicos e para-aórticos. Todas assinaram o consentimento pós-informado para a realização dos procedimentos de estadiamento cirúrgico com a linfadenectomia pélvica e para-aórtica. As pacientes foram tratadas no período de novembro de 1999 a maio de 2001 . O estudo foi aprovado pela Comissão de Ética em Pesquisa do Hospital das Clínicas da Faculdade de Medicina de Ribeirão Preto como parte de um projeto de avaliação de resultados de tratamento de pacientes com carcinoma avançado do colo do útero.

Todas foram submetidas a estadiamento de acordo com as normas da FIGO $^{15}$ : avaliação clínica, radiografia de tórax, urografia excretora, retossigmoidoscopia e cistoscopia com biópsia quando indicado. Foi feita avaliação da função renal e hepática para seleção de casos para quimioterapia. A média de idade das pacientes incluídas foi de 56,2 anos (40 a 73 anos). O índice de massa corporal (IMC) destas pacientes variou de 18 a 34. O grupo de pacientes não obesas (IMC igual ou inferior a 25) contava com 16 casos e o grupo de obesas (IMC maior que 25), com 20 pacientes. Quanto ao tipo histológico, 31 casos fo- 
ram classificados como epidermóides e cinco apresentavam componente de adenocarcinoma.

Previamente ao início da cirurgia, com a paciente anestesiada, realizava-se o toque sob anestesia para se avaliar a extensão da lesão para vagina e paramétrios e indícios de comprometimento tumoral dos órgãos vizinhos. O protocolo para o estadiamento cirúrgico previa a inspeção da superficie peritoneal e biópsia de lesões suspeitas, a avaliação das relações entre o tumor, bexiga e reto por palpação manual ou instrumental conforme a metodologia disponível. Tanto pela via laparoscópica como pela via laparotômica, iniciou-se a linfadenectomia pela ressecção do conteúdo perivascular e da fossa obturadora entre as iliacas externa e interna e sobre o músculo psoas. O ureter não foi descolado do peritôneo e marcou o limite medial da dissecção. Este conteúdo era submetido a exame intra-operatório de congelação, e caso algum gânglio fosse positivo procediase a linfadenectomia para-aórtica por via aberta. A abordagem dos grandes vasos foi feita sempre pelo lado direito, dissecando-se o ureter, veia cava inferior, artéria mesentérica inferior, aorta abdominal até a emergência das renais, tendo como alvo as cadeias pré-vasculares e intervasculares.

A linfadenectomia pélvica por via laparotômica foi realizada em 17 pacientes e por via laparoscópica em 19. Em duas destas 19 pacientes houve a conversão para laparotomia para se obter acesso adequado aos gânglios para-aórticos. A linfadenectomia para-aórtica completa foi realizada em 14 pacientes que apresentavam gânglios pélvicos positivos. Em outras duas, com evidência de comprometimento ganglionar extenso, realizouse a linfadenectomia para-aórtica seletiva. Em três pacientes a avaliação intra-operatória dos gânglios pélvicos foi negativa e a avaliação pela coloração $\mathrm{HE}$ foi positiva. Estas foram submetidas posteriormente a procedimento cirúrgico para linfadenectomia para-aórtica. A média de gânglios obtidos foi de 14,7 (faixa de 7 a 24) para linfonodos pélvicos e de 11,4 (8 a 18) para as cadeias para-aórticas.

Dos 36 casos incluídos, 34 foram submetidas a quimioterapia neoadjuvante com três ciclos de cisplatina $\left(50 \mathrm{mg} / \mathrm{m}^{2}\right)$ D1 e D2 e fluoruracil $\left(1000 \mathrm{mg} / \mathrm{m}^{2}\right) \mathrm{D} 1 \mathrm{a} \mathrm{D} 5$, repetidos a cada 28 dias após o estadiamento cirúrgico. Uma paciente estadiada cirurgicamente como IB1, foi submetida a cirurgia de Wertheim-Meigs como tratamento definitivo e uma outra paciente com invasão vesical comprovada por biópsia foi submetida a radioterapia exclusiva.

A análise constou da comparação da distribuição de estádios obtidos com o estadiamento clínico e o obtido com os achados durante a cirurgia inclusive a análise histológica dos gânglios e outras amostras (considerado padrão-ouro). Secundariamente foram analisadas alguns parâmetros relacionados à abordagem cirúrgica: duração dos procedimentos, tipo e freqüência das complicações intra e pós-operatórias. Foi calculada a sensibilidade e acurácia do exame intra-operatório para avaliação do estado dos linfonodos.

\section{Resultados}

A avaliação clínica inicial levou à seguinte distribuição por estádios: IB2 em 6 casos, IIA em 2 casos, IIB em 21 casos e IIIB nos 7 casos restantes. A avaliação cirúrgica revelou extensão da doença diferente daquela obtida pela avaliação clínica em 18 pacientes $(50 \%)$. Entre os casos com discordância entre os métodos, cinco pacientes tinham doença menos extensa do que a indicada pelo estadiamento clinico, 7 pacientes apresentavam doença mais extensa na pelve e 6 apresentaram linfonodos paraaórticos comprometidos. Entre as pacientes com estádio modificado para menor, dois casos classificados como IIB foram estadiados cirurgicamente como IB2 - um caso e outro como IB1 (tratado com cirurgia radical); um caso IIB foi reclassificado como IIA, um caso de IIIB para IIB e um caso passou de IIIB para IIIA. Nos casos de modificação do estadio para maior, dois casos IB2 foram reclassificadas como IIB, um caso inicialmente IIA para IIB, dois casos IIB que passaram para IIIB, um caso de IIB para IVA e um caso de IIIB para IVA. Dos 36 casos, $14(38,9 \%)$ apresentavam gânglios pélvicos com metástases. Entre as pacientes que apresentaram linfonodos para-aórticos positivos, quatro haviam sido classificadas no estadiamento cirúrgico como IIB e duas como IIIB (Tabela 1).

Tabela 1 - Correlação entre estadiamento clínico (FIGO)15 e o cirúrgico em 36 pacientes com carcinoma de colo avançado.

\begin{tabular}{|c|c|c|c|c|c|c|c|c|c|}
\hline $\begin{array}{l}\text { Estadiamento } \\
\text { clínico }\end{array}$ & IB1 & $\mathrm{IB}_{2}$ & IIA & IIB & IIIA & IIIB & IVA & $\begin{array}{l}\text { GG. } \\
\text { PA+ }\end{array}$ & \\
\hline IB2 & & 4 & & 2 & & & & & 6 \\
\hline$\| A$ & & & 1 & 1 & & & & & 2 \\
\hline IIB & 1 & 2 & 1 & 10 & & 2 & 1 & 4 & 21 \\
\hline IIIA & & & & & 0 & & & & - \\
\hline IIIB & & & & 1 & 1 & 2 & 1 & 2 & 7 \\
\hline IVA & & & & & & & 0 & & - \\
\hline IVB & & & & & & & & 0 & - \\
\hline Total & 1 & 6 & 2 & 14 & 1 & 4 & 2 & 6 & 36 \\
\hline
\end{tabular}


Ao estratificarmos estes resultados conforme o estadiamento clínico inicial, observamos que para os estádios clinicos mais iniciais as mudanças de estadiamento são mais infreqüentes. Para os estadios IB e IIA houve um estadiamento errôneo em dois dos sete casos avaliados $(28.6 \%)$, para o estadio IIB a diferença foi superior a metade dos 22 casos e no estadios IIIB houve subestadiamento em cinco dos sete casos.

A avaliação anatomopatológica no intra-operatório detectou 11 dos 14 casos com gânglios pélvicos positivos (três falso-negativos), conferindo ao método sensibilidade de $78,6 \%$ e especificidade de $100 \%$ (Tabela 2). A linfadenectomia paraaórtica revelou um grau de comprometimento ganglionar de $20 \%$ nos casos de linfonodos pélvicos positivos, para os casos de linfadenectomias completas. Se considerarmos também os casos de linfadenectomia seletiva, a positividade sobe para $33 \%$. Do total de casos $16,7 \%$ apresentavam gânglios para-aórticos positivos.

Tabela 2 - Correlação entre o avaliação histopatológica intra-operatória e o exame histopatológico em parafina, para os gânglios pélvicos de pacientes com carcinoma de colo avançado.

\begin{tabular}{lccc}
\hline $\begin{array}{l}\text { Gânglios } \\
\text { pélvicos }\end{array}$ & \multicolumn{3}{c}{$\begin{array}{c}\text { Exame histopatológico de congelação } \\
\text { intra-operatório }\end{array}$} \\
\hline Positivos & Positivos & Negativos & Total \\
Negativos & 11 & 3 & 14 \\
Total & 0 & 21 & 22 \\
\hline
\end{tabular}

A duração da linfadenectomia pélvica e paraaórtica teve média de $278 \mathrm{~min}$ (variando de 120 a 375 minutos). Já a execução da linfadenectomia pélvica apresentou duração média de 190 minutos (variando de 110 a 340 minutos) nos procedimentos realizados por laparotomia e 188 minutos (variando de 180 a 205 minutos) nos procedimentos por laparoscopia.

Houve complicações no intra-operatória em oito casos. Ocorreram sete casos de lesão traumática dos grandes vasos, sendo cinco lesões arteriais e duas venosas, todas tratadas com sutura simples e sem repercussão hemodinâmica ou seqüelas a longo prazo. Houve ainda lesão de ureter em uma paciente diagnosticada e tratada com sucesso no intra-operatório. Três pacientes necessitaram reposição com concentrado de hemácias. As três pacientes estavam incluídas no grupo submetido à dissecção para-aórtica.

As pacientes permaneceram internadas durante quatro dias em média (variação de 2 a 6), e após a alta hospitalar três delas foram readmitidas para tratamento de infecção de parede abdominal, controlada com antibioticoterapia e curativos locais.

\section{Discussão}

Há muito é conhecida a imprecisão do estadiamento clínico. Estudos nos quais se compara o desempenho do estadiamento clínico e o do cirúrgico, para todos os estádios, mostram divergências em porcentagem que varia de 25 a $33 \%$ dos $\operatorname{casos}^{16,17}$. Quando se considera apenas os estádios iniciais (IB e II), 20\% a $22 \%$ das mulheres no estadio IB e $39 \%$ a $44 \%$ das mulheres nos estadios II são subestadiadas clinicamente ${ }^{12,18}$. Entre as neoplasias ginecológicas, as do colo permanecem como as únicas cujo estadiamento não é cirúrgico o que implica em altas taxas de classificação incorreta especialmente o subestadiamento ${ }^{19}$. Além disto, a presença de gânglios com metástases não modifica o estádio previamente estabelecido o que contraria os conhecimentos acumulados a respeito da evolução das neoplasias. É fundamental, portanto, que a imprecisão do estadiamento seja reduzida.

As modificações do estadiamento pela exploração cirúrgica são devidas aos achados referentes aos paramétrios, invasão vesical ou retal e disseminação pela superfície peritoneal e gânglios. Neste estudo, devido à avaliação intra-operatória foram modificados o comprometimento parametrial e/ou vaginal para maior em 13 pacientes dos 36 casos (três casos de comprometimento vaginal e dez de extensão parametrial). Além destes, em dois casos foi diagnosticado comprometimento vesical, confirmados por biópsia, com avaliações prévias por cistoscopia negativas exceto pela presença de edema na região do trígono. Modificações em proporções semelhantes (entre 20 e $60 \%$ dos casos) foram observadas em outras séries e são tão mais freqüentes quanto mais elevado o estádio clínico prévio $^{20-22}$.

Estas informações tornam o estadiamento cirúrgico um passo necessário na propedêutica de avaliação das lesões avançadas de colo de útero para a escolha correta do tratamento.

Dos parâmetros citados, no entanto, o mais relevante é o estado dos gânglios pélvicos e paraaórticos pois, tanto para tumores iniciais como para os localmente avançados, a presença de metástases em linfonodos é o fator de prognóstico para a sobrevida mais importante ${ }^{23,24}$. Entre os casos nos estádios IB e II a presença de gânglios para-aórticos positivos isoladamente é estimada 
em $0,9 \%$ e um quarto das pacientes com linfonodos pélvicos positivos apresentarão metástases na cadeia para-aórtica ${ }^{20}$. Desta forma, para limitar a morbidade associada à linfadenectomia indicamos a dissecção das cadeias para-aórticas apenas para os casos com gânglios pélvicos positivos. O número de gânglios analisados é semelhante ao obtido em investigações similares; inferior ao obtido por Michel et al. ${ }^{20}$ (média de 34 ) e superior ao obtido por Kohler et al. ${ }^{24}$ (média de 23).

Como em outras casuísticas ${ }^{12,18}$ a proporção do comprometimento de linfonodos para-aórticos é crescente em relação ao estádio clínico (1 a $5 \%$ dos casos no estadio IB, $11 \%$, nos tumores IB2, superior a $15 \%$ no estadio IIB, e de $25 \%$ entre as pacientes com linfonodos pélvicos positivos ${ }^{20}$.

Na presença de linfonodos para-aórticos positivos as alternativas para tratamento são: a ressecção, o emprego de campos para-aórticos ou combinação de ambos. Os resultados dos estudos são discordantes. Em alguns estudos se observou melhora da sobrevida após a ressecção de gânglios considerados comprometidos na exploração cirúrgica $^{14,25}$. A inclusão dos campos para-aórticos na área irradiada é outra alternativa com ganho em sobrevida e intervalo livre de doença mas que está associada a aumento importante da taxa de complicações ${ }^{26,27}$.

Outra alternativa para estes casos, é o tratamento neoadjuvante com quimioterapia. Importante lembrar que a recomendação para tratamento dos casos de carcinoma avançado do colo com radioterapia associada a quimiossensibilização foi baseada em estudos nos quais foram incluídas pacientes submetidas ao estadiamento cirúrgico. No entanto os casos com linfonodos para-aórticos e, em um dos estudos também os pélvicos, comprometidos foram excluídos destes estudos ${ }^{5,28}$. Desta forma as conclusões destes estudos não são aplicáveis a pacientes com gânglios comprometidos. Para estes casos a melhor alternativa é o emprego de quimioterapia com doses completas.

Em resumo o estadiamento cirúrgico levou a mudança no planejamento de tratamento que seria adotado pelo estadiamento clínico em um quinto dos casos. Este resultado é semelhante ao obtido em outras investigações em que se incluiu pacientes com carcinoma localmente avançado $\mathrm{e}^{12}$ ou mesmo quando se considera o conjunto de pacientes com câncer de colo, inicial e avançado ${ }^{17}$.

Quanto à segurança do procedimento, observamos que a complicação mais freqüente foi lesão de grandes vasos. No entanto houve necessidade de reposição apenas em três casos (todos durante dissecção de linfonodos para-aórticos). Ocorreu infecção em 3 de 36 casos, também sem seqüelas. Os tipos e as porcentagens de complicações ocorridas são semelhantes aos de outros estudos semelhantes em que se empregou linfadenectomia transperitoneal ${ }^{29}$. O tempo cirúrgico prolongado (média de 278 minutos para linfadenectomia completa) é devido, parcialmente, à avaliação intra-operatória dos gânglios. Em outras séries em que se empregou a mesma via, o tempo total variou de 170 a 200 minutos $^{30,31}$. É de se prever que com o acúmulo de experiência o tempo cirúrgico médio diminua como se observa em outros estudos.

Concluímos que o estadiamento cirúrgico demanda experiência e requer disponibilidade para um procedimento de longa duração. No entanto, devido ao número de casos em que há modificações no estadiamento clínico inicial e portanto da conduta deve ser levado em consideração para as pacientes com tumores localmente avançados especialmente para investigação sobre resultados de tratamento.

\section{Referências}

1. Parkin DM, Bray F, Ferlay J, Pisani P. Global cancer statistics, 2002. CA Cancer J Clin. 2005;55(2):74-108.

2. Brasil. Ministério da Saúde. Secretaria de Atenção à Saúde. Instituto Nacional de Câncer. Coordenação de Prevenção e Vigilância. Estimativa 2005: incidência de câncer no Brasil. Rio de Janeiro: INCA; 2004.

3. Thuler LC, Mendonça GA. Estadiamento inicial dos casos de câncer de mama e colo do útero em mulheres brasileiras. Rev Bras Ginecol Obstet. 2005; 27(11): 656-60.

4. Morris M, Eifel PJ, Lu J, Grigsby PW, Levenback C, Stevens RE, et al. Pelvic radiation with concurrent chemotherapy compared with pelvic and para-aortic radiation for high-risk cervical cancer. N Engl J Med. 1999;340(15):1137-43.

5. Keys HM, Bundy BN, Stehman FB, Muderspach LI, Chafe WE, Suggs CL 3rd, et al. Cisplatin, radiation, and adjuvant hysterectomy compared with radiation and adjuvant hysterectomy for bulky stage IB cervical carcinoma. N Engl J Med. 1999;340(15):1154-61.

6. Piver MS, Lele SB, Malfetano JH. cisDiamminedichloroplatinum II based combination chemotherapy for the control of extensive paraaortic lymph node metastasis in cervical cancer. Gynecol Oncol. 1987;26(1):71-6.

7. Loizzi V, Cormio G, Loverro G, Selvaggi L, Disaia PJ, Cappuccini F. Chemoradiation: a new approach for the treatment of cervical cancer. Int $\mathrm{J}$ Gynecol Cancer. 2003;13(5):580-6. 
8. Neoadjuvant Chemotherapy for Cervical Cancer Meta-Analysis Collaboration (NACCCMA) Collaboration. Neoadjuvant chemotherapy for locally advanced cervix cancer. Cochrane Database Syst Rev. 2004;(2):CD001774.

9. Neoadjuvant Chemotherapy for Locally Advanced Cervical Cancer Meta-analysis Collaboration. Neoadjuvant chemotherapy for locally advanced cervical cancer: a systematic review and meta-analysis of individual patient data from 21 randomised trials. Eur J Cancer. 2003;39(17):2470-86.

10.Landoni F, Maneo A, Colombo A, Placa F, Milani R, Perego $\mathrm{P}$, et al. Randomised study of radical surgery versus radiotherapy for stage Ib-IIa cervical cancer. Lancet. 1997;350(9077):535-40.

11. Marnitz S, Kohler C, Roth C, Fuller J, Hinkelbein W, Schneider A. Is there a benefit of pretreatment laparoscopic transperitoneal surgical staging in patients with advanced cervical cancer? Gynecol Oncol. 2005;99(3):536-44.

12. Goff BA, Muntz HG, Paley PJ, Tamimi HK, Koh WJ, Greer BE. Impact of surgical staging in women with locally advanced cervical cancer. Gynecol Oncol. 1999;74(3):436-42.

13. Hertel H, Kohler C, Elhawary T, Michels W, Possover M, Schneider A. Laparoscopic staging compared with imaging techniques in the staging of advanced cervical cancer. Gynecol Oncol. 2002;87(1):46-51.

14. Cosin JA, Fowler JM, Chen MD, Paley PJ, Carson LF, Twiggs LB. Pretreatment surgical staging of patients with cervical carcinoma: the case for lymph node debulking. Cancer. 1998;82(11):2241-8.

15. Benedet JL, Bender H, Jones H 3rd, Ngan HY, Pecorelli S. FIGO staging classifications and clinical practice guidelines in the management of gynecologic cancers. FIGO Committee on Gynecologic Oncology. Int J Gynaecol Obstet. 2000;70(2):209-62.

16. Onnis A, Maggino T, Marchetti M. Surgery as diagnostic and therapeutic moment in the management of cervical cancer. Eur J Gynaecol Oncol. 1985;6(1):20-30.

17. Hasenburg A, Salama JK, Van TJ, Amosson C, Chiu JK, Kieback DG. Evaluation of patients after extraperitoneal lymph node dissection and subsequent radiotherapy for cervical cancer. Gynecol Oncol. 2002;84(2):321-6.

18. Podczaski ES, Palombo C, Manetta A, Andrews C, Larson J, DeGeest $K$, et al. Assessment of pretreatment laparotomy in patients with cervical carcinoma prior to radiotherapy. Gynecol Oncol. 1989;33(1):71-5.

19. Shepherd JH. Cervical and vulva cancer: changes in FIGO definitions of staging. Br J Obstet Gynaecol. 1996;103(5):405-6.

20. Michel G, Morice P, Castaigne D, Leblanc M, Rey A,
Duvillard P. Lymphatic spread in stage Ib and II cervical carcinoma: anatomy and surgical implications. Obstet Gynecol. 1998;91(3):360-3.

21. Holcomb K, Abulafia O, Matthews RP, Gabbur N, Lee YC, Buhl A. The impact of pretreatment staging laparotomy on survival in locally advanced cervical carcinoma. Eur J Gynaecol Oncol. 1999;20(2):90-3.

22. Fowler JM, Carter JR, Carlson JW, Maslonkowski R, Byers LJ, Carson LF, et al. Lymph node yield from laparoscopic lymphadenectomy in cervical cancer: a comparative study. Gynecol Oncol. 1993;51(2):187-92.

23. Lanciano RM, Martz K, Coia LR, Hanks GE. Tumor and treatment factors improving outcome in stage III-B cervix cancer. Int $\mathrm{J}$ Radiat Oncol Biol Phys. 1991;20(1):95-100.

24. Kohler C, Klemm P, Schau A, Possover M, Krause $\mathrm{N}$, Tozzi R, et al. Introduction of transperitoneal lymphadenectomy in a gynecologic oncology center: analysis of 650 laparoscopic pelvic and/or paraaortic transperitoneal lymphadenectomies. Gynecol Oncol. 2004;95(1):52-61.

25. Moore DH, Stehman FB. What is the appropriate management of early stage cervical cancer (International Federation of Gynecology and Obstetrics stages I and IIA), surgical assessment of lymph nodes, and role of therapeutic resection of lymph nodes involved with cancer? J Natl Cancer Inst Monogr. 1996;(21):43-6.

26. Stehman FB, Bundy BN, DiSaia PJ, Keys HM, Larson JE, Fowler WC. Carcinoma of the cervix treated with radiation therapy. I. A multi-variate analysis of prognostic variables in the Gynecologic Oncology Group. Cancer. 1991;67(11):2776-85.

27. Rotman M, Pajak TF, Choi K, Clery M, Marcial V, Grigsby PW, et al. Prophylactic extended-field irradiation of paraaortic lymph nodes in stages IIB and bulky IB and IIA cervical carcinomas. Ten-year treatment results of RTOG 79-20. JAMA. 1995;274(5):387-93.

28. Narayan K, McKenzie AF, Hicks RJ, Fisher R, Bernshaw D, Bau S. Relation between FIGO stage, primary tumor volume, and presence of lymph node metastases in cervical cancer patients referred for radiotherapy. Int J Gynecol Cancer. 2003;13(5):65763.

29. Rose PG, Bundy BN, Watkins EB, Thigpen JT, Deppe G, Maiman MA, et al. Concurrent cisplatin-based radiotherapy and chemotherapy for locally advanced cervical cancer. N Engl J Med. 1999;340(15):1144-53.

30. Renaud MC, Plante M, Roy M. Combined laparoscopic and vaginal radical surgery in cervical cancer. Gynecol Oncol. 2000;79(1):59-63.

31. Schlaerth JB, Spirtos NM, Carson LF, Boike G, Adamec $\mathrm{T}$, Stonebraker B. Laparoscopic retroperitoneal lymphadenectomy followed by immediate laparotomy in women with cervical cancer: a gynecologic oncology group study. Gynecol Oncol. 2002;85(1):81-8. 